the expected range of $0.11-2.27 / 100$ ventilator days ${ }^{1}$, we identified staffing levels and sedation as key areas which appeared to have some causal effect on these numbers. Following the audit, staff were made aware of the increase in UE's and the importance of prevention was highlighted.

Results Between May 2011 and March 2012. all incident forms were examined and those relating to 'accidental extubations' or 'tube displacement' were examined in more detail. During this time period it was noted that the UE rate was $0.65 / 100$ ventilator days. Conclusion Despite reviewing the sedation policy and staffing levels, the UE rate remains unchanged. It is possible that the heightened awareness of staff has increased the reporting rate but there is no evidence of this. Although our UE rate remains low we will continue to examine each case individually and review practice as necessary.

1. da Silva et al 2010. Pediatr Crit Care Med. Unplanned extubation in pediatric critically ill patients.

\section{RANDOMIZED CONTROLLED TRIAL ON EARLY L-CARNITINE SUPPLEMENTATION TO PRETERM NEWBORNS WITH RESPIRATORY DISTRESS SYNDROME. DOES IT INFLUENCE NEONATAL WELLBEING?}

doi:10.1136/archdischild-2012-302724.1003

R Said, M Abd El Meguid, D Mosallam, M Mahmoud, Neonatology. Pediatrics \& Neonatology, Faculty of Medicine Cairo University, Cairo, Egypt

Background and Aim Respiratory distress syndrome (RDS) is the most common cause of respiratory failure and requirement for mechanical ventilation (MV) of newborns. L-carnitine is essential for the processes underlying fetal maturation such as surfactant synthesis. This study aims to study the effect of early carnitine supplementation on respiratory, nutritional, hematological parameters in preterm neonates with respiratory distress syndrome.

Methods This study was conducted on 60 preterm infants 30-33 weeks of gestation presenting with RDS divided randomly into 2 groups: group A comprised 30 preterm infants who received I.V. L-carnitine at a dose of $30 \mathrm{mg} / \mathrm{Kg} /$ day until oral intake was established; then it was given orally at the same dose, group B comprised 30 preterm infants who did not receive L-carnitine. Laboratory and radiological investigations were done to confirm RDS, to evaluate and to follow up growth pattern, hematological, and respiratory outcome in those neonates.

Results L-Carnitine supplementation resulted in significant increase in weight gain, decreased period of stay on mechanical ventilator and duration of hospital stay as well as improved discharge status in group A.

Conclusion L-carnitine could be routinely used for preterm neonates suffering from RDS to reduce the need of mechanical ventilation and oxygen requirements in addition to its role in growth. However its effects on hematological parameters should be traced in further research work.

\section{THREE DIFFERENT MASK HOLDS FOR POSITIVE PRESSURE VENTILATION IN A NEONATAL MANNEQUIN}

doi:10.1136/archdischild-2012-302724.1004

'EV Wilson, 'JE O'Shea, 'M Thio, 1,2JA Dawson, ${ }^{2} \mathrm{R}$ Boland, 1,2.3PG Davis. 'Newborn Research, The Royal Women's Hospital; ${ }^{2}$ The Murdoch Childrens Research Institute, Parkville: ${ }^{3}$ University of Melbourne, Melbourne, VIC, Australia

Background and Aim Mask ventilation is commonly used for neonatal resuscitation. Variable leak and inconsistent tidal volumes are reported in mannequin and delivery room studies. We compared the spider hold ( $\mathrm{SH}$ ) against the two-point top hold (TPTH), and two-handed hold (THH) for delivering positive pressure ventilation (PPV).

Methods 53 participants from 5 professional groups provided PPV with each hold for 1 minute to a mannequin, using a T-piece resuscitator (PIP/PEEP 30/5 $\mathrm{cmH}_{2} \mathrm{O}, 40-60$ inflations/min). Mask leak and expired tidal volume $\left(\mathrm{T}_{\mathrm{VE}}\right)$ were measured with a flow sensor. ANOVA was used to compare the average median leak from each participant for each hold and by professional group.

Results 7324 inflations were analysed.

Abstract 1004 Table 1 Leak (\%) mean(SD)

\begin{tabular}{lccc}
\hline Professional Group & $\begin{array}{c}\text { TPTH } \mathbf{n}=\mathbf{2 5 5 4} \\
\text { inflations }\end{array}$ & $\begin{array}{c}\text { SH } \mathbf{n}=\mathbf{2 3 8 4} \\
\text { inflations }\end{array}$ & $\begin{array}{c}\text { THH } \mathbf{n = 2 4 0 6} \\
\text { inflations }\end{array}$ \\
\hline Consultant $\mathrm{n}=10$ & $34(28)$ & $39(40)$ & $4535)$ \\
Fellow $\mathrm{n}=10$ & $33(27)$ & $32(32)$ & $33(35)$ \\
Registrar $\mathrm{n}=10$ & $31(22)$ & $38(23)$ & $13(14)$ \\
Midwife $\mathrm{n}=12$ & $40(30)$ & $43(36)$ & $48(36)$ \\
Neonatal nurse $\mathrm{n}=11$ & $35(28)$ & $38(34)$ & $40(33)$ \\
\hline
\end{tabular}

The mean (SD) leak was 35(27)\%, 38(34)\% and 39(33)\% for the TPTH, SH and THH respectively ( $p=0.003)$. The mean (SD) $T_{V E}$ was not significantly different between the three holds $(p=0.09$ ). The lowest mean (SD) leak was measured with the THH by registrars $13(14) \%$ and highest by midwives with the THH 48(36)\% ( $\mathrm{p}=0.001)$.

Conclusion Each hold can be used to give PPV. The SH does not appear to reduce leak when compared to the other holds.

\section{CALCULATED INHALED ALBUTEROL DOSE AND DELIVERED ALBUTEROL DOSE DURING SIMULATED INFANT VENTILATION USING A NOVEL VENTILATOR CIRCUIT CONNECTOR}

doi:10.1136/archdischild-2012-302724.1005

'J Mazela, ${ }^{1} \mathrm{~K}$ Chmura, ${ }^{2} \mathrm{M}$ Kulza, ${ }^{2} \mathrm{E}$ Florek, ${ }^{3} \mathrm{TJ}$ Gregory, ${ }^{3} \mathrm{C}$ Henderson, ${ }^{4} \mathrm{M}$ Keszler. ${ }^{1}$ Neonatology; 'Laboratory of Environmental Research, Poznan University of Medical Sciences, Poznan, Poland; ${ }^{3}$ Research and Development, Discovery Laboratories, Inc.; ${ }^{4}$ Brown University, Women and Infants Hospital, Warrington, PA, USA

Background Dose determination of aerosolized medications administered to ventilated infants is based on nominal dose and not on the calculated target inhaled dose. A novel ventilator circuit connector (VCC) (AFECTAIR ${ }^{\circledR}$, Discovery Laboratories, Inc. Warrington, $\mathrm{PA}$ ), has been developed to facilitate inhaled therapies to ventilated patients.

Objective The aim of the study was to evaluate the difference between the calculated inhaled dose (ID) and the actual delivered dose (DD) in an in vitro simulated infant ventilation system using the VCC vs standard of care (SoC).

Design/methods Albuterol sulfate (AS) was aerosolized with a jet nebulizer and delivered using SoC and VCC. Aerosolized AS was collected on HME filters placed before a test lung under different ventilation conditions. DD was determined by rinsing the filters and using an HPLC assay for AS. The calculated ID was determined using the formula: $\mathrm{ID}=\mathrm{Ca} * \mathrm{Vm},(\mathrm{Ca}=$ aerosol concentration, $\mathrm{Vm}=\mathrm{m}$ inute ventilation).

Results There was a 10-14 fold increase in the in vitro DD of AS at various ventilation conditions when using the VCC compared with SoC. The difference between the calculated ID and the measured $\mathrm{DD}$ in vitro ranged from $-42 \%$ to $15 \%$ for the VCC and from $-388 \%$ to $-158 \%$ for SoC.

Conclusions The VCC delivered a higher AS dose in vitro that was more representative of the calculated ID compared with SoC. The 